\title{
A!
}

This is an electronic reprint of the original article.

This reprint may differ from the original in pagination and typographic detail.

Östergård, Patric R.J.; Paavola, William T.

\section{Mappings of Butson-type Hadamard matrices}

Published in:

Discrete Mathematics

DOI:

10.1016/j.disc.2018.05.012

Published: 01/09/2018

Document Version

Peer reviewed version

Published under the following license:

CC BY-NC-ND

Please cite the original version:

Östergård, P. R. J., \& Paavola, W. T. (2018). Mappings of Butson-type Hadamard matrices. Discrete Mathematics, 341(9), 2387-2397. https://doi.org/10.1016/j.disc.2018.05.012

This material is protected by copyright and other intellectual property rights, and duplication or sale of all or part of any of the repository collections is not permitted, except that material may be duplicated by you for your research use or educational purposes in electronic or print form. You must obtain permission for any other use. Electronic or print copies may not be offered, whether for sale or otherwise to anyone who is not an authorised user. 


\title{
Mappings of Butson-Type Hadamard Matrices ${ }^{\text {th }}$
}

\author{
Patric R. J. Östergård ${ }^{\mathrm{a}, *}$, William T. Paavola ${ }^{\mathrm{a}}$ \\ ${ }^{a}$ Aalto University School of Electrical Engineering \\ Department of Communications and Networking \\ P.O. Box 15400, 00076 Aalto, Finland
}

\begin{abstract}
A $\operatorname{BH}(q, n)$ Butson-type Hadamard matrix $H$ is an $n \times n$ matrix over the complex $q$ th roots of unity that fulfills $H H^{*}=n I_{n}$. It is well known that a $\mathrm{BH}(4, n)$ matrix can be used to construct a $\mathrm{BH}(2,2 n)$ matrix, that is, a real Hadamard matrix. This method is here generalised to construct a $\mathrm{BH}(q, p n)$ matrix from a $\mathrm{BH}(p q, n)$ matrix, where $q$ has at most two distinct prime divisors, one of them being $p$. Moreover, an algorithm for finding the domain of the mapping from its codomain in the case $p=q=2$ is developed and used to classify the $\mathrm{BH}(4,16)$ matrices from a classification of the $\mathrm{BH}(2,32)$ matrices.
\end{abstract}

Keywords: Butson-type Hadamard matrix, classification, complex matrix, monomial equivalence, mapping

\section{Introduction}

A $\operatorname{BH}(q, n)$ Butson-type Hadamard matrix $H$ is an $n \times n$ matrix with entries that are complex $q$ th roots of unity, such that $H H^{*}=n I_{n}$ where $H^{*}$ denotes the conjugate transpose of $H$ and $I_{n}$ is the $n \times n$ identity matrix [1]. Butson-type Hadamard matrices generalise (real) Hadamard matrices, which are $\mathrm{BH}(2, n)$ matrices. For more information about Hadamard matrices in general and Butson-type Hadamard matrices in particular, see, for example, $[6,15,18]$.

An interesting open problem in this area is the Hadamard Conjecture, which asserts that $\mathrm{BH}(2, n)$ matrices exist whenever $n$ is divisible by 4 . With respect to this conjecture, the $\mathrm{BH}(4, n)$ matrices have also received

\footnotetext{
Supported in part by the Academy of Finland, Grant No. 289002.

${ }^{*}$ Corresponding author

Email addresses: patric.ostergard@aalto.fi (Patric R. J. Östergård), william.paavola@aalto.fi (William T. Paavola)
} 
attention. The following theorem is [2, Theorem 1]; Turyn [19] gives credit to Williamson [20] for some of the underlying theory.

Theorem 1. If there is a $\mathrm{BH}(4, n)$ matrix, then there is a $\mathrm{BH}(2,2 n)$ matrix.

If the Complex Hadamard Conjecture - saying that $\mathrm{BH}(4, n)$ matrices exist whenever $n$ is divisible by 2 -is true, then Theorem 1 implies that the Hadamard Conjecture is also true. (The name of this conjecture comes from the fact that $\mathrm{BH}(4, n)$ matrices have been called complex Hadamard matrices and quaternary complex Hadamard matrices in the literature.)

An increasing interest in $\mathrm{BH}(q, n)$ matrices with $q \notin\{2,4\}$ has raised the question whether there are results similar to that in Theorem 1 for other values of $q$. One such result was obtained by Compton, Craigen, and de Launey [3].

Theorem 2. If there is a $\mathrm{BH}(6, n)$ matrix with no entries in $\{-1,1\}$, then there is a $\mathrm{BH}(2,4 n)$ matrix.

Theorem 2 can further be seen as a corollary of Theorem 1 and the following result from [13].

Theorem 3. If there is a $\mathrm{BH}(6, n)$ matrix with no entries in $\{-1,1\}$, then there is a $\mathrm{BH}(4,2 n)$ matrix.

Further work on generalizing these theorems has been carried out by Egan and Ó Catháin [5].

In this paper, we generalise Theorem 1 and show that a $\mathrm{BH}(q, p n)$ matrix can be constructed from a $\mathrm{BH}(p q, n)$ matrix, where $q$ is divisible by at most two distinct prime numbers, and $p$ is one of them. This is the topic of Section 2. In Section 3, we discuss equivalence of Butson-type Hadamard matrices, especially in the context of the results of Section 2. In Section 4, we consider the computational problem of finding the domain of the mapping considered in Section 2 when $p=q=2$. Specifically, we develop an algorithm for classifying the $\mathrm{BH}(4, n)$ matrices from a classification of the $\mathrm{BH}(2,2 n)$ matrices, and apply this to the classification of the $\mathrm{BH}(2,32)$ matrices carried out by Kharaghani and Tayfeh-Rezaie [8, 9]. These results corroborate the computational classification of $\mathrm{BH}(4,16)$, obtained recently in [13] using different techniques.

\section{Mappings of Matrices}

We denote the $(i, j)$ th entry of a matrix $M$ by $M_{i j}$ and the set of $q$ th roots of unity by

$$
\Omega_{q}:=\left\{\omega \in \mathbb{C}: \omega^{q}=1\right\} .
$$


Some types of mappings for Butson-type Hadamard matrices are well known and have been thoroughly studied; perhaps the most basic and natural one is the Kronecker product.

Definition 1. Let $A$ be $a \times n$ matrix and $B$ a square matrix. The Kronecker product of $A$ and $B$ is defined as

$$
A \otimes B=\left[\begin{array}{ccc}
A_{11} B & \ldots & A_{1 n} B \\
\vdots & & \vdots \\
A_{n 1} B & \ldots & A_{n n} B
\end{array}\right] .
$$

Lemma 1. $(A \otimes B)(C \otimes D)=A C \otimes B D$ whenever $A C$ and $B D$ are defined.

It is easy to show that the Kronecker product of a $\mathrm{BH}(q, n)$ matrix and a $\mathrm{BH}\left(q, n^{\prime}\right)$ matrix is a $\mathrm{BH}\left(q, n n^{\prime}\right)$ matrix. In this straightforward mapping only the matrix dimensions change. We shall now consider mappings from $\operatorname{BH}(q, n)$ matrices to $\operatorname{BH}\left(q^{\prime}, n^{\prime}\right)$ matrices, where $q^{\prime}<q$. For this we need some definitions.

Definition 2. The filtering function $f_{q}: \mathbb{C} \rightarrow \Omega_{q} \cup\{0\}$ is

$$
f_{q}(x):= \begin{cases}x & \text { if } x \in \Omega_{q} \\ 0 & \text { otherwise }\end{cases}
$$

When applied to a matrix $H, f_{q}(H)$ acts elementwise. For a given root of unity $\zeta$, we further define $F_{k, q, \zeta}(H):=f_{q}\left(\zeta^{k} H\right)$.

Lemma 2. Let $q=p^{a} r^{b}, a \geq 1, b \geq 0$, where $p$ and $r$ are primes. If $H$ is a $\operatorname{BH}(p q, n)$ matrix and $\zeta \in \Omega_{p q} \backslash \Omega_{q}$, then $H=\sum_{k=0}^{p-1} \zeta^{-k} F_{k, q, \zeta}(H)$.

Proof. The (multiplicative) cyclic group $G$ over $\Omega_{q}$ is a subgroup of the (multiplicative) cyclic group $G^{\prime}$ over $\Omega_{p q}$. The result follows if we can show that for an arbitrary element $a \in \Omega_{p q}$, the elements $a \zeta^{0}, a \zeta^{1}, \ldots, a \zeta^{p-1}$ form a transversal of the cosets of $G$ in $G^{\prime}$, whereby exactly one of the elements is in $\Omega_{q}$. Indeed, if $a \zeta^{i}$ and $a \zeta^{j}$ with $0 \leq i<j \leq p-1$ would belong to the same coset, then we would have $\zeta^{j-i} \in \Omega_{q}$, and then $\zeta \in \Omega_{q(j-i)}$. However, since $(j-i)$ and $p$ share no prime divisors, we have $\Omega_{p q} \cap \Omega_{q(j-i)}=\Omega_{q}$, so this would further imply that $\zeta \in\left(\Omega_{p q} \backslash \Omega_{q}\right) \cap \Omega_{q(j-i)}=\emptyset$, a contradiction.

Definition 3. Let $R_{m, p, \zeta}:=R_{p, \zeta} \otimes I_{m / p}$, where $m$ is divisible by $p$ and $R_{p, \zeta}$ is the $p \times p$ monomial matrix

$$
\left[\begin{array}{ccccc}
0 & 0 & \cdots & 0 & \zeta^{-p} \\
1 & 0 & \cdots & 0 & 0 \\
0 & 1 & \cdots & 0 & 0 \\
& & \ddots & & \\
0 & 0 & \cdots & 1 & 0
\end{array}\right] .
$$


Proving the following properties of the $m \times m$ matrix $R_{m, p, \zeta}$ is a matter of direct calculation. Similar properties hold for $R_{p, \zeta}$.

Lemma 3. $R_{m, p, \zeta} R_{m, p, \zeta}^{*}=I_{m}$ and $R_{m, p, \zeta}^{p}=\zeta^{-p} I_{m}$.

Definition 4. For a $\mathrm{BH}(p q, n)$ matrix $H$, a $\mathrm{BH}(q, m)$ matrix $C$, and $\zeta \in$ $\Omega_{p q} \backslash \Omega_{q}$, let

$$
L(H, C, \zeta):=\sum_{k=0}^{p-1} R_{m, p, \zeta}^{k} C \otimes F_{k, q, \zeta}(H) .
$$

Next, we will show that $L(H, C, \zeta)$ is a Butson-type Hadamard matrix by using minimal vanishing sums.

Definition 5. A summation of roots of unity is said to be a vanishing sum if the sum is 0 . A vanishing sum whose summands cannot be partitioned into two nonempty vanishing sums is called a minimal vanishing sum.

Lemma 4. [11, Corollary 3.4] Let $q=p^{a} r^{b}$, where $p$ and $r$ are primes, and let $\omega_{p}$ and $\omega_{r}$ be primitive pth and rth roots of unity, respectively. Then, up to multiplying each summand with a fixed element from $\Omega_{q}$, the minimal vanishing sums of qth roots of unity are exactly

$$
1+\omega_{p}+\omega_{p}^{2}+\cdots+\omega_{p}^{p-1}=0, \quad 1+\omega_{r}+\omega_{r}^{2}+\cdots+\omega_{r}^{r-1}=0 .
$$

Lemma 5. Consider summations over $\Omega_{p q}$, where $q=p^{a} r^{b}$ with $p$ and $r$ prime and $a \geq 1, b \geq 0$. For $0 \leq k \leq p-1$, let $S_{k}$ be a summation of qth roots of unity, and let $\zeta \in \Omega_{p q} \backslash \Omega_{q}$. If $\sum_{k=0}^{p-1} \zeta^{k} S_{k}=0$, then $S_{k}=0$ for $0 \leq k \leq p-1$.

Proof. Consider the case $b \geq 1$. The summation $\sum_{k=0}^{p-1} \zeta^{k} S_{k}=0$ consists of summands from $\Omega_{p q}$, so by Lemma 4 , its minimal vanishing sums contain the $p$ th roots of unity, the $r$ th roots of unity, or are obtained by multiplying the summands of these with some element of $\Omega_{p q}$. The $p$ th and $r$ th roots of unity are in $\Omega_{q}$, and $\zeta^{k} \Omega_{q}, 0 \leq k \leq p-1$ forms a partition of $\Omega_{p q}$. Hence for any given minimal vanishing sum, there is a $k, 0 \leq k \leq p-1$, such that all summands are in $\zeta^{k} \Omega_{q}$. For all $k, 0 \leq k \leq p-1$, the summands of $\zeta^{k} S_{k}$ are from $\zeta^{k} \Omega_{q}$, so for any given minimal vanishing sum of $\sum_{k=0}^{p-1} \zeta^{k} S_{k}=0$, there is a $k, 0 \leq k \leq p-1$, such that all summands are from $\zeta^{k} S_{k}$. Then for each $k, 0 \leq k \leq p-1, \zeta^{k} S_{k}$ can be written as a sum of vanishing sums, so $\zeta^{k} S_{k}=0$ and $S_{k}=0$.

The case $b=0$ follows similarly; then there is no vanishing sum of $r$ th roots of unity. 
We will need the following formula for the product of two sums.

\section{Lemma 6.}

$$
\begin{aligned}
\sum_{k=0}^{p-1} f(k) \sum_{l=0}^{p-1} g(l)= & \sum_{k=0}^{p-1} f(k) g(k) \\
& +\sum_{u=1}^{p-1}\left(\sum_{k=0}^{p-1-u} f(k) g(u+k)+\sum_{k=p-u}^{p-1} f(k) g(u-p+k)\right) .
\end{aligned}
$$

Theorem 4. Let $q=p^{a} r^{b}, a \geq 1, b \geq 0$, where $p$ and $r$ are primes. If there is a $\mathrm{BH}(p q, n)$ matrix and $a \mathrm{BH}(q, m)$ matrix, where $m$ is divisible by $p$, then there is a $\mathrm{BH}(q, n m)$ matrix.

Proof. Let $H$ be a $\mathrm{BH}(p q, n)$ matrix and $C$ a $\operatorname{BH}(q, m)$ matrix, and let $\zeta \in$ $\Omega_{p q} \backslash \Omega_{q}$. We shall show that $L(H, C, \zeta)$ is a $\operatorname{BH}(q, n m)$ matrix.

Since $q, \zeta$, and $H$ are fixed, to improve readability we omit these parameters in expressions of the form $F_{k, q, \zeta}(H)$ and simply write $F_{k}$. Using Lemmas 2 and 6, we get

$$
\begin{aligned}
H H^{*} & =\sum_{k=0}^{p-1} \zeta^{-k} F_{k} \sum_{l=0}^{p-1} \zeta^{l} F_{l}^{*} \\
& =\sum_{k=0}^{p-1} F_{k} F_{k}^{*}+\sum_{u=1}^{p-1}\left(\sum_{k=0}^{p-u-1} \zeta^{-k} F_{k} \zeta^{u+k} F_{u+k}^{*}+\sum_{k=p-u}^{p-1} \zeta^{-k} F_{k} \zeta^{u-p+k} F_{u-p+k}^{*}\right) \\
& =\sum_{k=0}^{p-1} F_{k} F_{k}^{*}+\sum_{u=1}^{p-1} \zeta^{u}\left(\sum_{k=0}^{p-u-1} F_{k} F_{u+k}^{*}+\zeta^{-p} \sum_{k=p-u}^{p-1} F_{k} F_{u-p+k}^{*}\right)
\end{aligned}
$$

Since $H H^{*}=n I_{n}$, each off-diagonal entry in the matrix of the final expression is a vanishing sum of $p q$ th roots of unity. In addition, on the diagonal we have

$$
\left(\sum_{k=0}^{p-1} F_{k} F_{k}^{*}\right)_{i i}=\sum_{j=1}^{n} \sum_{k=0}^{p-1}\left|\left(F_{k}\right)_{i j}\right|=n,
$$

since at any entry $F_{k} \neq 0$ for exactly one value of $k, 0 \leq k \leq p-1$, as shown in the proof of Lemma 2. Now subtract $n$ from the entries on the diagonal to arrive at the following equations for the diagonal and off-diagonal entries, 
respectively:

$$
\begin{array}{r}
\sum_{u=1}^{p-1} \zeta^{u}\left(\sum_{k=0}^{p-u-1} F_{k} F_{u+k}^{*}+\zeta^{-p} \sum_{k=p-u}^{p-1} F_{k} F_{u-p+k}^{*}\right)=0, \\
\sum_{k=0}^{p-1} F_{k} F_{k}^{*}+\sum_{u=1}^{p-1} \zeta^{u}\left(\sum_{k=0}^{p-u-1} F_{k} F_{u+k}^{*}+\zeta^{-p} \sum_{k=p-u}^{p-1} F_{k} F_{u-p+k}^{*}\right)=0 .
\end{array}
$$

Applying Lemma 5 to the two cases now gives

$\sum_{k=0}^{p-1} F_{k} F_{k}^{*}=n I_{n}, \quad \sum_{k=0}^{p-u-1} F_{k} F_{u+k}^{*}+\zeta^{-p} \sum_{k=p-u}^{p-1} F_{k} F_{u-p+k}^{*}=\mathbf{0}_{n}, \quad 1 \leq u \leq p-1$,

where $\mathbf{0}_{n}$ is the $n \times n$ all-zero matrix. Using Definition 4 and Lemmas 1,3 , and 6 , we then get

$$
\begin{aligned}
& L(H, C, \zeta) L(H, C, \zeta)^{*}=\left(\sum_{k=0}^{p-1} R_{m, p, \zeta}^{k} C \otimes F_{k}\right)\left(\sum_{l=0}^{p-1} C^{*}\left(R_{m, p, \zeta}^{*}\right)^{l} \otimes F_{l}^{*}\right) \\
& =\left(\sum_{k=0}^{p-1} R_{m, p, \zeta}^{k} \otimes F_{k}\right)\left(C C^{*} \otimes I_{n}\right)\left(\sum_{l=0}^{p-1} R_{m, p, \zeta}^{-l} \otimes F_{l}^{*}\right) \\
& =m\left(\sum_{k=0}^{p-1} R_{m, p, \zeta}^{k} \otimes F_{k}\right)\left(\sum_{l=0}^{p-1} R_{m, p, \zeta}^{-l} \otimes F_{l}^{*}\right) \\
& =m\left(\sum_{k=0}^{p-1} R_{m, p, \zeta}^{k-k} \otimes F_{k} F_{k}^{*}+\sum_{u=1}^{p-1}\left(\sum_{k=0}^{p-u-1} R_{m, p, \zeta}^{-u} \otimes F_{k} F_{u+k}^{*}+\sum_{k=p-u}^{p-1} R_{m, p, \zeta}^{p-u} \otimes F_{k} F_{u-p+k}^{*}\right)\right) \\
& =m I_{m} \otimes \sum_{k=0}^{p-1} F_{k} F_{k}^{*}+m \sum_{u=1}^{p-1}\left(\sum_{k=0}^{p-u-1} R_{m, p, \zeta}^{-u} \otimes F_{k} F_{u+k}^{*}+\sum_{k=p-u}^{p-1} \zeta^{-p} R_{m, p, \zeta}^{-u} \otimes F_{k} F_{u-p+k}^{*}\right) \\
& =m I_{m} \otimes n I_{n}+m \sum_{u=1}^{p-1} R_{m, p, \zeta}^{-u} \otimes\left(\sum_{k=0}^{p-u-1} F_{k} F_{u+k}^{*}+\zeta^{-p} \sum_{k=p-u}^{p-1} F_{k} F_{u-p+k}^{*}\right) \\
& =n m I_{n m}+m \sum_{u=1}^{p-1} R_{m, p, \zeta}^{-u} \otimes \mathbf{0}_{n}=n m I_{n m} .
\end{aligned}
$$

As the entries of the $n m \times n m$ matrix $L(H, C, \zeta)$ are in $\Omega_{q}$, it is indeed a $\mathrm{BH}(q, n m)$ matrix.

Corollary 1. Let $q=p^{a} r^{b}, a \geq 1, b \geq 0$, where $p$ and $r$ are primes. If there is a $\mathrm{BH}(p q, n)$ matrix, then there is a $\mathrm{BH}(q, p n)$ matrix. 
Proof. For every prime $p$, there exists a $\mathrm{BH}(p, p)$ matrix, the Fourier matrix. Since $p$ divides $q$, such a matrix is also a $\mathrm{BH}(q, p)$ matrix, which can be used as $C$ in Theorem 4 .

In Corollary 1 , setting $p=q=2$ gives Theorem 1 , the well-known mapping from quaternary complex Hadamard matrices to real Hadamard matrices.

Note that the general construction cannot be extended by changing the requirement that the parameter $q$ of the original $\mathrm{BH}(p q, n)$ matrix be divisible by $p$. For example, there is a $\mathrm{BH}(6,2)$ matrix - take a $\mathrm{BH}(2,2)$ real matrixbut no $\mathrm{BH}(3,4)$ matrix [11, Theorem 5.2].

Example 1. We will now illustrate the results by constructing a $\mathrm{BH}(3,9)$ matrix from a $\mathrm{BH}(9,3)$ matrix; then we have $p=3, q=3$, and $n=3$ in Corollary 1. We let $\zeta=e^{\mathbf{i} 2 \pi / 9}$ and express elements in the log form (using exponents of $\zeta$ ) for clarity with just empty space for the element 0 . Now $C$ is the $3 \times 3$ Fourier matrix and $C_{k}:=R_{3,3, \zeta}^{k} C$, where $0 \leq k \leq 2$, are as follows:

$$
C_{0}=\left[\begin{array}{lll}
0 & 0 & 0 \\
0 & 3 & 6 \\
0 & 6 & 3
\end{array}\right], \quad C_{1}=\left[\begin{array}{lll}
6 & 3 & 0 \\
0 & 0 & 0 \\
0 & 3 & 6
\end{array}\right], \quad C_{2}=\left[\begin{array}{lll}
6 & 0 & 3 \\
6 & 3 & 0 \\
0 & 0 & 0
\end{array}\right] .
$$

Now, writing $F_{k}$ instead of $F_{k, q, \zeta}(H), C_{0} \otimes F_{0}+C_{1} \otimes F_{1}+C_{2} \otimes F_{2}$ is the desired mapping.

Consider the $\mathrm{BH}(9,3)$ matrix

$$
H=\left[\begin{array}{lll}
1 & 1 & 2 \\
2 & 5 & 0 \\
0 & 6 & 4
\end{array}\right]
$$

Then we get

$$
F_{0}=\left[\begin{array}{lll} 
& & 0 \\
0 & 6 &
\end{array}\right], \quad F_{1}=\left[\begin{array}{lll}
3 & 6 & 3 \\
& &
\end{array}\right], \quad F_{2}=\left[\begin{array}{lll}
3 & 3 & \\
& & 6
\end{array}\right],
$$

and the final $\mathrm{BH}(3,9)$ matrix is

$$
\left[\begin{array}{lllllllll}
0 & 0 & 0 & 3 & 3 & 6 & 6 & 6 & 3 \\
0 & 3 & 0 & 6 & 0 & 0 & 3 & 6 & 0 \\
0 & 6 & 3 & 0 & 6 & 6 & 0 & 6 & 0 \\
0 & 0 & 3 & 6 & 6 & 3 & 3 & 3 & 3 \\
3 & 6 & 0 & 3 & 6 & 3 & 3 & 6 & 6 \\
0 & 6 & 3 & 3 & 0 & 0 & 6 & 3 & 6 \\
3 & 3 & 3 & 3 & 3 & 6 & 3 & 3 & 0 \\
3 & 6 & 0 & 6 & 0 & 6 & 0 & 3 & 3 \\
0 & 6 & 6 & 6 & 3 & 6 & 3 & 0 & 6
\end{array}\right] .
$$


Considering elements in $\Omega_{3}$ rather than $\Omega_{9}$, the matrix takes the form

$$
\left[\begin{array}{lllllllll}
0 & 0 & 0 & 1 & 1 & 2 & 2 & 2 & 1 \\
0 & 1 & 0 & 2 & 0 & 0 & 1 & 2 & 0 \\
0 & 2 & 1 & 0 & 2 & 2 & 0 & 2 & 0 \\
0 & 0 & 1 & 2 & 2 & 1 & 1 & 1 & 1 \\
1 & 2 & 0 & 1 & 2 & 1 & 1 & 2 & 2 \\
0 & 2 & 1 & 1 & 0 & 0 & 2 & 1 & 2 \\
1 & 1 & 1 & 1 & 1 & 2 & 1 & 1 & 0 \\
1 & 2 & 0 & 2 & 0 & 2 & 0 & 1 & 1 \\
0 & 2 & 2 & 2 & 1 & 2 & 1 & 0 & 2
\end{array}\right] .
$$

\section{Equivalence of Hadamard Matrices}

Two Butson-type Hadamard matrices in $\operatorname{BH}(q, n)$ are said to be monomially equivalent if one can be obtained from the other by a sequence of row and column permutations, and multiplications of rows and columns by some $q$ th roots of unity. Moreover, in the definition of Hadamard equivalent matrices, one further considers application of a fixed automorphism of the multiplicative cyclic group with elements in $\Omega_{q}$. Either of these equivalence relations partitions the $\mathrm{BH}(q, n)$ matrices into equivalence classes. In the classification of (Butson-type) Hadamard matrices, the aim is to find a transversal of the equivalence classes.

In the sequel, we specifically consider monomial equivalence of matrices and write $H \cong H^{\prime}$ when $H$ and $H^{\prime}$ are equivalent, that is, if there exist monomial matrices $P$ and $Q$ such that $P H Q=H^{\prime}$.

In the context of classification, it would be particularly useful if equivalence of $H$ and $H^{\prime}$ would imply equivalence of $L(H, C, \zeta)$ and $L\left(H^{\prime}, C, \zeta\right)$. Then, to find the equivalence classes in the image of $L$, we would only have to compute $L(H, C, \zeta)$ for one $H$ in each equivalence class of $\mathrm{BH}(p q, n)$. Unfortunately, it turns out that $L$ does not always preserve monomial equivalence.

Example 2. Let $p=2, q=4$, and $\zeta=e^{\mathbf{i} 2 \pi / 8}$, and consider

$$
\begin{aligned}
& C=\left[\begin{array}{rr}
1 & 1 \\
1 & -1
\end{array}\right], \quad R_{2,2, \zeta} C=\left[\begin{array}{rr}
-\mathbf{i} & \mathbf{i} \\
1 & 1
\end{array}\right] . \\
& H=\left[\begin{array}{rr}
1 & 1 \\
1 & -1
\end{array}\right], \quad H^{\prime}=\left[\begin{array}{rr}
1 & \zeta \\
1 & -\zeta
\end{array}\right] \cong H .
\end{aligned}
$$

Then

$$
L(H, C, \zeta)=\left[\begin{array}{rrrr}
1 & 1 & 1 & 1 \\
1 & -1 & 1 & -1 \\
1 & 1 & -1 & -1 \\
1 & -1 & -1 & 1
\end{array}\right], \quad L\left(H^{\prime}, C, \zeta\right)=\left[\begin{array}{rrrr}
1 & 1 & 1 & -1 \\
1 & -1 & 1 & 1 \\
1 & \mathbf{i} & -1 & \mathbf{i} \\
1 & -\mathbf{i} & -1 & -\mathbf{i}
\end{array}\right]
$$


There is no sequence of row and column operations that transforms $L\left(H^{\prime}, C, \zeta\right)$ into a matrix with only real elements, so $L(H) \varsubsetneqq L\left(H^{\prime}\right)$ even though $H \cong H^{\prime}$.

In some special cases, however, $L$ does preserve monomial equivalence, and this observation will be used in Section 4 to obtain a classification of the $\mathrm{BH}(4,16)$ matrices. In fact, equivalence is always preserved for all operations except column multiplication. For column multiplication, a sufficient condition for equivalence to be preserved is that, given $C$ and $\zeta$, there exist a monomial matrix $M$ such that $C=R_{m, p, \zeta} C M$. (Finding Butson-type Hadamard matrices $C$ for which the condition can be fulfilled seems difficult in general.) To show this, we first consider a property of the filtering function $f_{q}$.

Lemma 7. Let $H$ be a $\mathrm{BH}(p q, n)$ matrix. If $P$ and $Q$ are $n \times n$ monomial matrices whose nonzero entries are from $\Omega_{q}$, then $f_{q}(P H Q)=P f_{q}(H) Q$.

Proof. Write $P=P^{\prime} D$, where $P$ is a permutation matrix and $D$ is a diagonal matrix with diagonal entries from $\Omega_{q}$. Assume that $P^{\prime}$ maps the $i^{\prime}$ th row to the $i$ th row when multiplied from the left. Then

$$
\begin{aligned}
\left(P f_{q}(H)\right)_{i j} & =\left(P^{\prime} D f_{q}(H)\right)_{i j}=\left(D f_{q}(H)\right)_{i^{\prime} j}=D_{i^{\prime} i^{\prime}} f_{q}(H)_{i^{\prime} j} \\
& = \begin{cases}D_{i^{\prime} i^{\prime}} H_{i^{\prime} j} & \text { if } H_{i^{\prime} j} \in \Omega_{q}, \\
0 D_{i^{\prime} i^{\prime}} & \text { otherwise. }\end{cases} \\
& = \begin{cases}D_{i^{\prime} i^{\prime}} H_{i^{\prime} j} & \text { if } D_{i^{\prime} i^{\prime}} H_{i^{\prime} j} \in \Omega_{q}, \\
0 & \text { otherwise. }\end{cases} \\
& = \begin{cases}\left(P^{\prime} D H\right)_{i j} & \text { if }\left(P^{\prime} D H\right)_{i j} \in \Omega_{q}, \\
0 & \text { otherwise. }\end{cases} \\
& =f_{q}(P H)_{i j} .
\end{aligned}
$$

When multiplying from the right, we similarly get $\left(f_{q}(H) Q\right)_{i j}=f_{q}(H Q)_{i j}$, so $f_{q}(P H Q)=P f_{q}(H Q)=P f_{q}(H) Q$.

The following result is proved in [7, Corollary 4.3.10].

Lemma 8. If $A_{1}, \ldots, A_{k}$ are $m \times m$ matrices and $B_{1}, \ldots, B_{k}$ are $n \times n$ matrices, then

$$
A_{1} \otimes B_{1}+\cdots+A_{k} \otimes B_{k} \cong B_{1} \otimes A_{1}+\cdots+B_{k} \otimes A_{k} .
$$


Theorem 5. Let $q=p^{a} r^{b}, a \geq 1, b \geq 0$, where $p$ and $r$ are primes. Let $H$ and $H^{\prime}$ be $\mathrm{BH}(p q, n)$ matrices, let $C$ be a $\mathrm{BH}(q, m)$ matrix where $m$ is divisible by $p$, and let $\zeta \in \Omega_{p q} \backslash \Omega_{q}$. If $C=R_{m, p, \zeta} C M$ for some monomial matrix $M$ and $H \cong H^{\prime}$, then $L(H, C, \zeta) \cong L\left(H^{\prime}, C, \zeta\right)$.

Proof. Consider first row and column permutations and multiplication by elements of $\Omega_{q}$, leaving only multiplication by elements of $\Omega_{p q}$ to be considered later. Let $H^{\prime}:=P H Q$, where $P$ and $Q$ are $n \times n$ monomial matrices whose nonzero entries are from $\Omega_{q}$. We now get

$$
\begin{aligned}
L\left(H^{\prime}, C, \zeta\right) & =\sum_{k=0}^{p-1} R_{m, p, \zeta}^{k} C \otimes f_{q}\left(\zeta^{k} H^{\prime}\right) \\
& =\sum_{k=0}^{p-1} R_{m, p, \zeta}^{k} C \otimes f_{q}\left(\zeta^{k} P H Q\right) \\
& =\sum_{k=0}^{p-1} R_{m, p, \zeta}^{k} C \otimes P f_{q}\left(\zeta^{k} H\right) Q \\
& =\left(I_{m} \otimes P\right)\left(\sum_{k=0}^{p-1} R_{m, p, \zeta}^{k} C \otimes f_{q}\left(\zeta^{k} H\right)\right)\left(I_{m} \otimes Q\right) \\
& =\left(I_{m} \otimes P\right) L(H, C, \zeta)\left(I_{m} \otimes Q\right),
\end{aligned}
$$

where Lemma 7 is used in the third row. The matrices $I_{m} \otimes P$ and $I_{m} \otimes Q$ are monomial, so $L\left(H^{\prime}, C, \zeta\right) \cong L(H, C, \zeta)$.

Consider now multiplication of the first row by $\zeta$. Since the opposite order of the matrices in the Kronecker product is more convenient in the sequel, we first use Lemma 8 to show that

$$
L(H, C, \zeta)=\sum_{k=0}^{p-1} R_{m, p, \zeta}^{k} C \otimes F_{k, q, \zeta}(H) \cong \sum_{k=0}^{p-1} F_{k, q, \zeta}(H) \otimes R_{m, p, \zeta}^{k} C .
$$

Similarly, $L\left(H^{\prime}, C, \zeta\right) \cong \sum_{k=0}^{p-1} F_{k, q, \zeta}\left(H^{\prime}\right) \otimes R_{m, p, \zeta}^{k} C$.

The function $F_{k, q, \zeta}$ can also be applied to a $k \times n$ matrix $M$ that is a submatrix of $H$ whereby $\sum_{k=0}^{p-1} F_{k, q, \zeta}(M) \otimes R_{m, p, \zeta}^{k} C$ is a submatrix of $\sum_{k=0}^{p-1} F_{k, q, \zeta}(H) \otimes$ $R_{m, p, \zeta}^{k} C$. Hence, considering all but the first $m$ rows, $\sum_{k=0}^{p-1} F_{k, q, \zeta}\left(H^{\prime}\right) \otimes$ $R_{m, p, \zeta}^{k} C$ and $\sum_{k=0}^{p-1} F_{k, q, \zeta}(H) \otimes R_{m, p, \zeta}^{k} C$ coincide. Let $h$ and $\zeta h$ be the first row of $H$ and $H^{\prime}$, respectively. The first $m$ rows of $\sum_{k=0}^{p-1} F_{k, q, \zeta}\left(H^{\prime}\right) \otimes R_{m, p, \zeta}^{k} C$ are 


$$
\begin{aligned}
& \sum_{k=0}^{p-1} F_{k, q, \zeta}(\zeta h) \otimes R_{m, p, \zeta}^{k} C \\
& =\sum_{k=0}^{p-1} F_{k+1, q, \zeta}(h) \otimes R_{m, p, \zeta}^{k} C \\
& =\sum_{k=1}^{p} F_{k, q, \zeta}(h) \otimes R_{m, p, \zeta}^{k-1} C \\
& =\left(I_{1} \otimes R_{m, p, \zeta}^{-1}\right) \sum_{k=1}^{p} F_{k, q, \zeta}(h) \otimes R_{m, p, \zeta}^{k} C \\
& =R_{m, p, \zeta}^{-1}\left(F_{p, q, \zeta}(h) \otimes R_{m, p, \zeta}^{p} C+\sum_{k=1}^{p-1} F_{k, q, \zeta}(h) \otimes R_{m, p, \zeta}^{k} C\right) \\
& =R_{m, p, \zeta}^{-1}\left(\zeta^{p} F_{0, q, \zeta}(h) \otimes \zeta^{-p} R_{m, p, \zeta}^{0} C+\sum_{k=1}^{p-1} F_{k, q, \zeta}(h) \otimes R_{m, p, \zeta}^{k} C\right) \\
& =R_{m, p, \zeta}^{-1} \sum_{k=0}^{p-1} F_{k, q, \zeta}(h) \otimes R_{m, p, \zeta}^{k} C \\
& \simeq \sum_{p-1}^{p} F_{k, q, \zeta}(h) \otimes R_{m, p, \zeta}^{k} C .
\end{aligned}
$$

The final expression equals the first $m$ rows of $\sum_{k=0}^{p-1} F_{k, q, \zeta}(H) \otimes R_{m, p, \zeta}^{k} C$. Importantly, we have equivalence in the last step via row operations only. Hence

$L(H, C, \zeta) \cong \sum_{k=0}^{p-1} F_{k, q, \zeta}(H) \otimes R_{m, p, \zeta}^{k} C \cong \sum_{k=0}^{p-1} F_{k, q, \zeta}\left(H^{\prime}\right) \otimes R_{m, p, \zeta}^{k} C \cong L\left(H^{\prime}, C, \zeta\right)$.

The fact that an arbitrary element $\omega \in \Omega_{p q}$ can be written as $\omega=\omega^{\prime} \zeta^{i}$, where $\omega^{\prime} \in \Omega_{q}$ and $0 \leq i \leq p-1$, can be used to handle the case of multiplication of the first row by $\omega$. The case of multiplication of other rows than the first one can be handled via a sequence of row permutations and multiplication of the first row.

Similar arguments hold for column multiplication. However, the multiplication of the first column by $\zeta$ might not give equivalent matrices, depending on $C$. Let $h$ and $\zeta h$ be the first columns of $H$ and $H^{\prime}$, respectively. If there exists a monomial matrix $M$ such that $C=R_{m, p, \zeta} C M$, then the first $m$ columns of $\sum_{k=0}^{p-1} F_{k, q, \zeta}\left(H^{\prime}\right) \otimes R_{m, p, \zeta}^{k} C$ are 


$$
\begin{aligned}
\sum_{k=0}^{p-1} F_{k, q, \zeta}(\zeta h) \otimes R_{m, p, \zeta}^{k} C & =\left(\sum_{k=1}^{p} F_{k, q, \zeta}(h) \otimes R_{m, p, \zeta}^{k}\right) R_{m, p, \zeta}^{-1} C \\
& =\left(\sum_{k=0}^{p-1} F_{k, q, \zeta}(h) \otimes R_{m, p, \zeta}^{k}\right) C M \\
& \cong \sum_{k=0}^{p-1} F_{k, q, \zeta}(h) \otimes R_{m, p, \zeta}^{k} C .
\end{aligned}
$$

Now, the submatrices are equivalent under column operations only, and again $L(H, C, \zeta) \cong L\left(H^{\prime}, C, \zeta\right)$.

\section{Finding the Domain of a Mapping}

Corollary 1 is about mappings of $\mathrm{BH}(q, n)$ matrices to $\mathrm{BH}\left(q^{\prime}, n^{\prime}\right)$ matrices where $q^{\prime}<q$ and $n^{\prime}>n$. A natural question is whether it is possible to do the reverse, that is, to construct $\mathrm{BH}(q, n)$ matrices from $\mathrm{BH}\left(q^{\prime}, n^{\prime}\right)$ matrices where $q^{\prime}<q$ and $n^{\prime}>n$. Although this is not always possible, one may take a computational approach and exhaustively search for the domain of a given mapping when the codomain is known.

In this section, we specifically consider finding the domain of

$$
L(\cdot, C, \zeta): \mathrm{BH}(4, n) \rightarrow \mathrm{BH}(2,2 n), \quad C=\left[\begin{array}{rr}
1 & 1 \\
-1 & 1
\end{array}\right], \quad \zeta=\mathbf{i} .
$$

This mapping also has the nice property that its image is easily described, as shown by Miyamoto [17]. (Note that there are two Lemma 4 in [17].)

Lemma 9. [17, Lemma 4, p. 104] There is a $\mathrm{BH}(2,2 n)$ matrix $M$ in block form $\left[\begin{array}{rr}A & B \\ -B & A\end{array}\right]$ if and only if there is a $\mathrm{BH}(4, n)$ matrix $H=\frac{1-\mathbf{i}}{2}(A+\mathbf{i} B)$.

Proof. In this proof we show both that Miyamoto's construction is the same as ours using the mapping $L(H, C, \zeta)$ in $(1)$, which proves the "if" case, and that any block form gives a matrix $H$, proving the "only if" case.

Let $H$ be a given $\mathrm{BH}(4, n)$ matrix. Write $F_{k, q, \zeta}(H)$ as $F_{k}$. Then

$$
L(H, C, \zeta)=\left[\begin{array}{rr}
1 & 1 \\
-1 & 1
\end{array}\right] \otimes F_{0}+\left[\begin{array}{rr}
1 & -1 \\
1 & 1
\end{array}\right] \otimes F_{1}=\left[\begin{array}{rr}
F_{0}+F_{1} & F_{0}-F_{1} \\
-\left(F_{0}-F_{1}\right) & F_{0}+F_{1}
\end{array}\right] .
$$

By letting $A=F_{0}+F_{1}$ and $B=F_{0}-F_{1}, L(H, C, \zeta)$ gets the desired block form and, by the proof of Theorem 4 , is a $\operatorname{BH}(2,2 n)$ matrix. Furthermore,

$$
H=F_{0}-\mathbf{i} F_{1}=\frac{A+B}{2}-\mathbf{i} \frac{A-B}{2}=\frac{1}{2}(A+\mathbf{i} B)-\frac{\mathbf{i}}{2}(A+\mathbf{i} B)=\frac{1-\mathbf{i}}{2}(A+\mathbf{i} B) .
$$


Starting from the matrix $M$, we get

$$
M M^{*}=\left[\begin{array}{rr}
A A^{*}+B B^{*} & -A B^{*}+B A^{*} \\
-B A^{*}+A B^{*} & B B^{*}+A A^{*}
\end{array}\right] .
$$

Since $M M^{*}=2 n I_{2 n}$, we then get $A A^{*}+B B^{*}=2 n I_{n}$ and $A B^{*}-B A^{*}=\mathbf{0}_{n}$. Now let $H=\frac{1-\mathbf{i}}{2}(A+\mathbf{i} B)$ to get

$$
H H^{*}=\frac{1}{2}\left(A A^{*}-\mathbf{i} A B^{*}+\mathbf{i} B A^{*}+B B^{*}\right)=n I_{n} .
$$

To prove that $H$ is a $\mathrm{BH}(4, n)$ matrix, we must still show that the entries of $H$ are in $\Omega_{4}$. This indeed holds, as we may write $H=\frac{1}{2}(A+B)-\frac{\mathbf{i}}{2}(A-B)$, and at any position $(i, j)$ exactly one of $A_{i j}+B_{i j}$ and $A_{i j}-B_{i j}$ is 0 and the other is -2 or 2 .

Note that when constructing a $\mathrm{BH}(2,2 n)$ matrix from a $\mathrm{BH}(4, n)$ matrix $H$, having $H=\frac{1-\mathbf{i}}{2}(A+\mathbf{i} B)$ is not a restriction; any $\mathrm{BH}(4, n)$ may be written in this form. Lemma 9 simplifies an exhaustive search for the domain of the mapping, as one may focus on finding the block forms $\left[\begin{array}{rr}A & B \\ -B & A\end{array}\right]$, which directly give the corresponding $\mathrm{BH}(4, n)$ matrices. Lemma 9 will play a central role in the sequel.

\subsection{Algorithm for Finding the Domain}

Using the parameters of (1), $C=R_{2,2, \zeta} C R_{2,2, \zeta}^{*}$, so $L(H, C, \zeta)$ preserves equivalence by Theorem 5 . We now consider $L$ to be a mapping from one set of equivalence classes to another:

$$
L(\cdot, C, \zeta):(\mathrm{BH}(4, n) / \cong) \rightarrow(\mathrm{BH}(2,2 n) / \cong)
$$

The mapping $L$ can be surjective for certain parameters, demonstrated by $(\mathrm{BH}(4,10) / \cong) \rightarrow(\mathrm{BH}(2,20) / \cong)$ and $(\mathrm{BH}(4,12) / \cong) \rightarrow(\mathrm{BH}(2,24) / \cong)$, but it is not surjective for $(\mathrm{BH}(4,14) / \cong) \rightarrow(\mathrm{BH}(2,28) / \cong)$ [14]. It turns out that it is not surjective for $(\mathrm{BH}(4,16) / \cong) \rightarrow(\mathrm{BH}(2,32) / \cong)$ either.

Since $L$ is neither surjective nor injective in general, it does not have an inverse. However, we may still consider the problem of finding all (equivalence classes of) Butson-type Hadamard matrices in the domain from those in the codomain. As we know of no analytical method for this, we approach the problem computationally. We will next develop an algorithm based on Lemma 9 and use it to classify the $\mathrm{BH}(4,16)$ matrices from a classification of the $\mathrm{BH}(2,32)$ matrices. 
Any $\mathrm{BH}(4, n)$ matrix is equivalent to a matrix where all entries of the first row and column are 1; such a matrix is called normalised. A normalised $n \times n$ matrix $H$ has $L(H, C, \zeta)$ in the block form

$$
L(H, C, \zeta)=\left[\begin{array}{rr}
A & B \\
-B & A
\end{array}\right], A=\left[\begin{array}{ll}
1 & \mathbf{1}^{\mathrm{T}} \\
\mathbf{1} & A_{s}
\end{array}\right], B=\left[\begin{array}{ll}
1 & \mathbf{1}^{\mathrm{T}} \\
\mathbf{1} & B_{s}
\end{array}\right]
$$

where $A_{s}$ and $B_{s}$ are $(n-1) \times(n-1)$ matrices and $\mathbf{1}$ is an $(n-1)$-dimensional vector of $1 \mathrm{~s}$.

Given a $\mathrm{BH}(2,2 n)$ matrix $M$, the aim is to search for all matrices $M^{\prime}$ such that $M^{\prime} \cong M$ and $M^{\prime}$ has the form (3). By Lemma 9, for each such $M^{\prime}$, there exists a $\mathrm{BH}(4, n)$ matrix $H$ for which $L(H, C, \zeta)=M^{\prime}$. The equivalence classes of $\mathrm{BH}(2,2 n)$ which are not in the image of $L$ and hence produce no $\mathrm{BH}(4, n)$ matrices are precisely those which do not contain any matrix in block form (3). In the sequel, we index the rows and columns from 0 to $2 n-1$.

We present a general algorithm as Algorithm 1. Algorithm 1 is essentially an exhaustive algorithm for finding all possible ways of transforming $H$ into the desired block form using equivalence-preserving operations.

Let us next consider some ways of reducing the search space and the size of the search tree.

Lemma 10. One choice of $i$ in line 1 suffices.

Proof. We shall show that we can choose $i=0$. A permutation of rows in $H$ is in one-to-one correspondence with permutations of pairs of rows $(j, n+j)$, $0 \leq j \leq n-1$, in $L(H, C, \zeta)$. Hence any choice of $i$ such that $1 \leq i \leq n-1$ gives solutions that are equivalent to solutions for $i=0$. On the other hand, by multiplying $H$ by the imaginary unit $\mathbf{i}$, so that $H^{\prime}:=\mathbf{i} H$, we get

$$
\begin{aligned}
L\left(H^{\prime}, C, \zeta\right) & =\left[\begin{array}{rr}
F(\mathbf{i} H)+F\left(\mathbf{i}^{2} H\right) & F(\mathbf{i} H)-F\left(\mathbf{i}^{2} H\right) \\
-F(\mathbf{i} H)+F\left(\mathbf{i}^{2} H\right) & F(\mathbf{i} H)+F\left(\mathbf{i}^{2} H\right)
\end{array}\right] \\
& =\left[\begin{array}{rr}
-B & A \\
-A & -B
\end{array}\right] \cong\left[\begin{array}{rr}
A & B \\
-B & A
\end{array}\right],
\end{aligned}
$$

where in the last step rows are permuted by swapping rows $j$ and $n+j$ for all $0 \leq j \leq n-1$. Hence, any choice of $i$ such that $0 \leq i \leq n-1$ gives solutions that are equivalent to solutions for $n \leq i \leq 2 n-1$.

The proof of the next lemma is similar to that of Lemma 10.

Lemma 11. One choice of $l$ in line 10 suffices. 


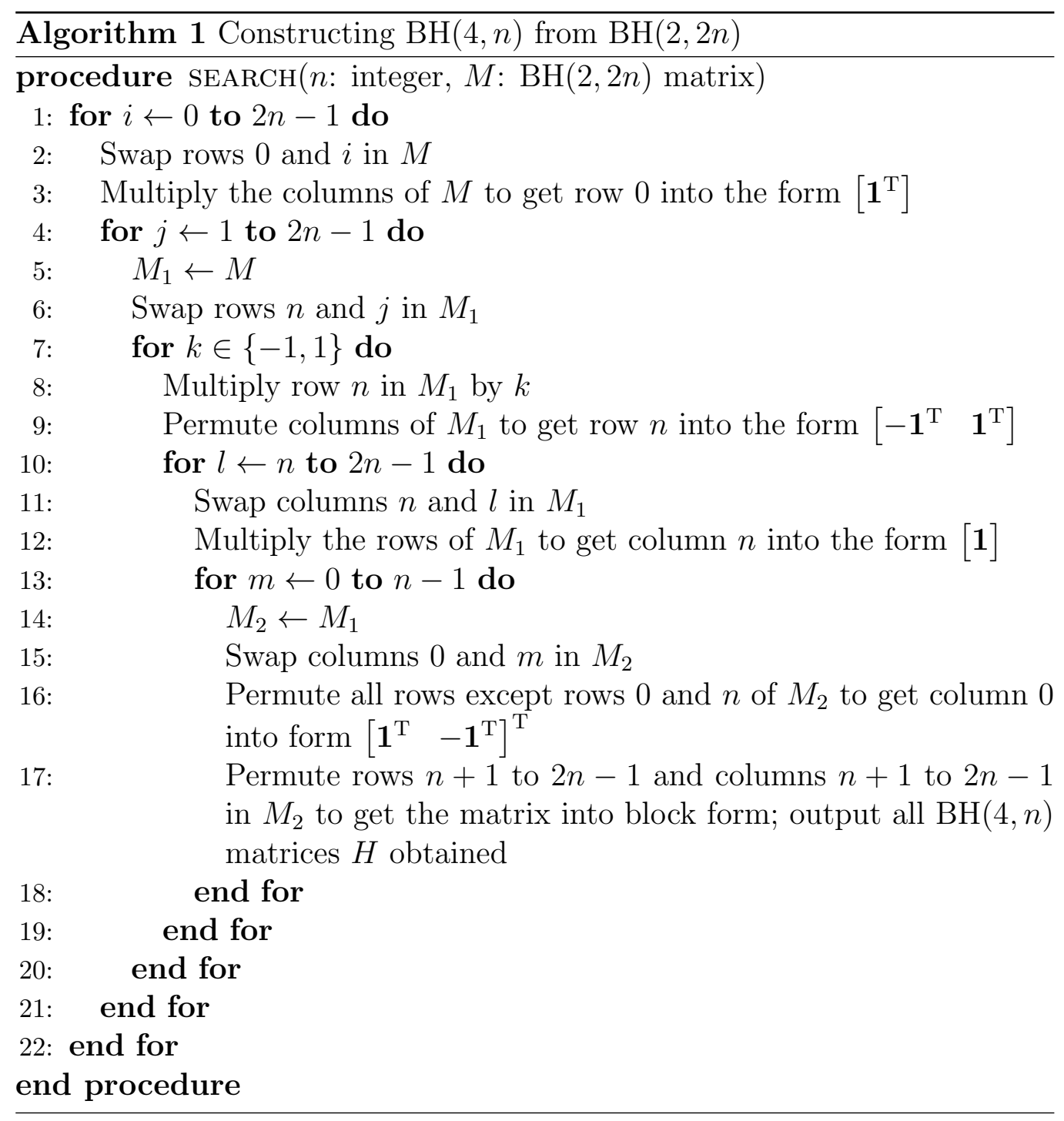


Consequently, the search tree branches only in lines 4, 7, and 13. But there is branching also within the subtask of line 17 , and this is actually the most time-consuming part of the algorithm. When reaching line 17, a matrix has the form

$$
\left[\begin{array}{rrrr}
1 & \mathbf{1}^{\top} & 1 & \mathbf{1}^{\top} \\
\mathbf{1} & A_{s} & \mathbf{1} & C \\
-1 & -\mathbf{1}^{\top} & 1 & \mathbf{1}^{\top} \\
-\mathbf{1} & D & \mathbf{1} & E
\end{array}\right]
$$

which should be transformed into

$$
\left[\begin{array}{rrrr}
1 & \mathbf{1}^{\top} & 1 & \mathbf{1}^{\top} \\
\mathbf{1} & A_{s} & \mathbf{1} & B_{s} \\
-1 & -\mathbf{1}^{\top} & 1 & \mathbf{1}^{\top} \\
-\mathbf{1} & -B_{s} & \mathbf{1} & A_{s}
\end{array}\right]
$$

with proper row and column permutations. The task is essentially to find all isomorphisms of $E$ into $A_{s}$ under row and column permutations, such that these permutations map $C$ and $D$ into matrices that are negations of each other. This can be done in several ways. One may, for example, find all required isomorphisms of $E$ into $A_{s}$, and for each such isomorphism check whether the transformations of $C$ and $D$ are as required. We are here dealing with a subtask that is frequently encountered in the study of equivalence of combinatorial objects [10]. To find isomorphisms, a tailored backtrack algorithm or the nauty [16] program may be employed.

The solutions that Algorithm 1 produces from a given $\mathrm{BH}(2,2 n)$ matrix still have to be checked for equivalence. We did this using nauty and a graph construction presented in [12], as discussed in [14]. Indeed, by Theorem 5, $\mathrm{BH}(4, n)$ matrices obtained from inequivalent $\mathrm{BH}(2,2 n)$ matrices cannot be equivalent.

The concept of Hadamard equivalence is related to the choice of value in line 7 of Algorithm 1. The multiplicative group with elements in $\Omega_{4}$ has an automorphism group of order 2 and corresponds to the complex conjugate.

Lemma 12. Up to complex conjugation, the two choices in line 7 give the same set of matrices $H$.

Proof. Consider $L(H, C, \zeta)$ for some specific $H$. By multiplying rows $n$ to $2 n-1$ by -1 and permuting columns, we get the following matrix equivalent to $L(H, C, \zeta)$ :

$$
L\left(H^{\prime}, C, \zeta\right)=\left[\begin{array}{rr}
B & A \\
-A & B
\end{array}\right]
$$


Now, using Lemma 9,

$$
H^{\prime}=\frac{1}{2}(B+A)-\frac{\mathbf{i}}{2}(B-A)=\overline{\frac{1}{2}(A+B)-\frac{\mathbf{i}}{2}(A-B)}=\bar{H},
$$

where $\bar{H}$ denotes the matrix with complex conjugated entries of $H$. As we multiplied rows $n$ to $2 n-1$ by -1 , this means that row $n$ was multiplied by -1 . Hence, there is a one-to-one correspondence between the matrices obtained for the respective choices of $k$ in line 7 of Algorithm 1, where the correspondence is given by complex conjugation of the entries of a matrix.

If Lemma 12 is used and monomial equivalence is considered in the classification, then for each $\mathrm{BH}(4, n)$ matrix obtained we have to construct an additional matrix by taking the complex conjugate of all entries.

\subsection{Results}

The presented algorithm was used in the current work to validate earlier classifications [14] of $\mathrm{BH}(4, n)$ matrices for $n \leq 14$. In particular, it was applied to the classification of the $\mathrm{BH}(2,32)$ matrices $[8,9]$ to obtain a classification of the $\mathrm{BH}(4,16)$ matrices. There are 13,710,027 equivalence classes of $\mathrm{BH}(2,32)$ matrices and the algorithm produced $1,786,763$ equivalence classes of $\mathrm{BH}(4,16)$ matrices from those. Recently, an alternative classification of the $\operatorname{BH}(4,16)$ matrices has been obtained in [13]; not only the numbers of objects but also the representatives obtained in both studies were checked and the results agree. This also gives further evidence of correctness of the classification of the $\mathrm{BH}(2,32)$ matrices.

It should finally be noted that the original classifications of the $\mathrm{BH}(2,32)$ and $\mathrm{BH}(4,16)$ matrices took tens of core years of computing time, whereas Algorithm 1 needed merely 24 core days to classify the latter matrices from the former.

\section{Acknowledgments}

The authors thank Ferenc Szöllősi for useful discussions.

\section{References}

[1] A. T. Butson, Generalized Hadamard matrices, Proc. Amer. Math. Soc. 13 (1962), 894-898.

[2] J. H. E. Cohn, Hadamard matrices and some generalisations, Amer. Math. Monthly 72 (1965), 515-518. 
[3] B. Compton, R. Craigen, and W. de Launey, Unreal $\mathrm{BH}(n, 6)$ 's and Hadamard matrices, Des. Codes Cryptogr. 79 (2016), 219-229.

[4] R. Craigen and H. Kharaghani, Orthogonal designs, in: C. J. Colbourn and J. H. Dinitz (Eds.), Handbook of Combinatorial Designs, 2nd ed., Chapman \& Hall/CRC, Boca Raton, 2007, pp. 280-295.

[5] R. Egan and P. Ó Catháin, Morphisms of Butson classes, preprint at arXiv:1707.08815.

[6] K. J. Horadam, Hadamard Matrices and Their Applications, Princeton University Press, Princeton, 2007.

[7] R. A. Horn and C. R. Johnson, Topics in Matrix Analysis, Cambridge University Press, Cambridge, 1991.

[8] H. Kharaghani and B. Tayfeh-Rezaie, On the classification of Hadamard matrices of order 32, J. Combin. Des. 18 (2010), 328-336.

[9] H. Kharaghani and B. Tayfeh-Rezaie, Hadamard matrices of order 32, J. Combin. Des. 21 (2013), 212-221.

[10] P. Kaski and P. R. J. Östergård, Classification Algorithms for Codes and Designs, Springer, Berlin, 2006.

[11] T. Y. Lam and K. H. Leung, On vanishing sums of roots of unity, $J$. Algebra 224 (2000), 91-109.

[12] P. H. J. Lampio and P. R. J. Östergård, Classification of difference matrices over cyclic groups, J. Statist. Plann. Inference 141 (2011), $1194-1207$.

[13] P. H. J. Lampio, P. R. J. Östergård, and F. Szöllősi, Orderly generation of Butson Hadamard matrices, preprint at arXiv:1707.02287.

[14] P. H. J. Lampio, F. Szöllősi, and P. R. J. Östergård, The quaternary complex Hadamard matrices of orders 10, 12, and 14, Discrete Math. 313 (2013), 189-206.

[15] W. de Launey, Generalized Hadamard matrices, in: C. J. Colbourn and J. H. Dinitz (Eds.), Handbook of Combinatorial Designs, 2nd ed., Chapman \& Hall/CRC, Boca Raton, 2007, pp. 301-306.

[16] B. D. McKay and A. Piperno, Practical graph isomorphism, II, J. Symbolic Comput. 60 (2014), 94-112. 
[17] M. Miyamoto, A construction of Hadamard matrices, J. Combin. Theory Ser. A 57 (1991), 86-108.

[18] J. Seberry and M. Yamada, Hadamard matrices, sequences, and block designs, in: J. H. Dinitz and D. R. Stinson (Eds.), Contemporary Design Theory: A Collection of Surveys, Wiley, New York, 1992, pp. 431560 .

[19] R. J. Turyn, Complex Hadamard matrices, in R. Guy, H. Hanani, N. Sauer, and J. Schonheim (Eds.), Combinatorial Structures and Their Applications, Gordon and Breach, New York, 1970, pp. 435-437.

[20] J. Williamson, Hadamard's determinant theorem and the sum of four squares, Duke Math. J. 11 (1944), 65-81. 